\title{
Anisotropic bending and twisting behaviour of a twin crystal composed of a diarylethene
}

\author{
Takuya Higashiguchi, Daichi Kitagawa, Seiya Kobatake
}

\begin{tabular}{|c|l|}
\hline Citation & CrystEngComm. 23(34); 5795-5800 \\
\hline Issue Date & $2021-09-14$ \\
\hline Published & $2020-12-28$ \\
\hline Type & Journal Article \\
\hline Textversion & author \\
\hline $\begin{array}{c}\text { Supporting } \\
\text { Information }\end{array}$ & $\begin{array}{l}\text { Supplementary information, movie and Crystal structure data. } \\
\text { Supplementary files is available at https://doi.org/10.1039/D0CE01705A. }\end{array}$ \\
\hline Relation & $\begin{array}{l}\text { The following article has been accepted by CrystEngComm. } \\
\text { This is the accepted manuscript version. } \\
\text { After it is published, it will be found at https://doi.org/10.1039/D0CE01705A. }\end{array}$ \\
\hline DOI & $10.1039 / D 0 C E 01705 \mathrm{~A}$ \\
\hline
\end{tabular}

\author{
Self-Archiving by Author(s) \\ Placed on: Osaka City University Repository
}

Higashiguchi, T., Kitagawa, D., \& Kobatake, S. (2021). Anisotropic bending and twisting behaviour of a twin crystal composed of a diarylethene. CrystEngComm. 23, 5795-5800. 


\section{Anisotropic bending and twisting behaviour of a twin crystal composed of a diarylethene}

Received 00th January 20xx, Accepted 00th January 20xx DOI: $10.1039 / \times 0 \times x 00000 x$

\author{
Takuya Higashiguchi, Daichi Kitagawa and Seiya Kobatake*
}

Here, we found that crystals consisting of 1,2-bis(2-methyl-5-(4-undecyloxyphenyl)-3-thienyl)perfluorocyclopentene (1a) exhibit unusual photomechanical bending. Upon irradiation with ultraviolet (UV) light, the crystal bent toward the incident light, but the velocity of the bending depended on the illumination directions. When irradiated from the left and right sides of the crystal, the velocity of the bending was different from each other. We found that the crystal is a "twin crystal", as confirmed by X-ray crystallography. The bending mechanism of the twin crystal can be interpreted by the content of twin components, the absorption anisotropy of diarylethene molecules, and the degree of the crystal twisting.

\section{Introduction}

Photomechanical materials efficiently transform light energy directly into mechanical energy even at extremely low temperatures, in water, and under vacuum. In particular, the photomechanical crystals have a potential to be used as functional actuators driven by photon energy for the next generation. Photomechanical crystals convert microscopic size changes in molecular structure associated with photoisomerization into macroscopic shape changes of a crystal. These crystals have higher Young's modulus than general polymer materials because the molecules are densely and regularly arranged. Furthermore, there is no contact with oxygen in a crystal, and photodegradation can be suppressed. Photoresponsive crystals with excellent durability are expected to be applied to photoactuators in microscopic areas such as nano wire, molecular motors, and soft robotics. ${ }^{1-3}$

Light can easily and remotely supply energy regardless of any amount. From such a point of view, representative photochromic molecules reacting even in the crystalline phase have been reported so far, such as diarylethene, ${ }^{4-20}$ azobenzene, ${ }^{21-25}$ salicylideneaniline, ${ }^{26,27}$ furylfulgide, ${ }^{28}$ anthracene ${ }^{2,29-32}$ and naphthalene diimides. ${ }^{33}$ In particular, the diarylethene crystals have the only photochromic properties such as excellent thermal stability, fast responsible time and high photoreversibility. ${ }^{34}$ Photoinduced shape changes of diarylethene crystals exhibit expansion and contraction, ${ }^{4,8}$ bending, ${ }^{5-11}$ curing, ${ }^{16}$ fragmentation ${ }^{17}$ and twisting. ${ }^{12,15}$ From a viewpoint of application to microactuators, the most useful motion is bending. Therefore, a mathematical perspective of the photomechanical crystal bending 35,36 and practical

Department of Applied Chemistry, Graduate School of Engineering, Osaka City University, 3-3-138 Sugimoto, Sumiyoshi-ku, Osaka 558-8585, Japan.

†Electronic Supplementary Information (ESI) available: X-ray crystallographic data and photograph of crystal $\mathbf{1 a}$, absorption anisotropy of $\mathbf{1 a}$ and $\mathbf{1 b}$ in crystal $\mathbf{1 a}$, photoinduced bending behaviour, and video of the bending behaviour. See DOI: $10.1039 / x 0 x x 00000 x$ mesoscopic photoactuators consisting of crystals of diarylethene derives ${ }^{5-7}$ have been reported so far. Although most of the deformation of diarylethene crystals is limited to simple bending, many researchers try to find new photomechanical motions. There are two main approaches. One strategy is the synthesis of new compounds and the investigation of photomechanical behaviour. In recent years, our group has reported molecular crystals that show stepwise bending ${ }^{13}$ and reciprocating bending ${ }^{14}$ by a combination of a photochromic reaction and a reversible single-crystal-to-singlecrystal phase transition. The other strategy is the management of light irradiation conditions. Irradiation conditions such as wavelength, ${ }^{9}$ intensity ${ }^{10}$ and polarization ${ }^{11}$ are deeply correlated with the photomechanical motions. Furthermore, by controlling the illumination direction, a single diarylethene crystal exhibits multiple photomechanical motions. ${ }^{12}$ However, these are all single crystals, and no one has focused on the shape of the crystals because it is difficult to intentionally control the crystal shape.

Here, we report on a photomechanical behaviour of a twin crystal composed of 1,2-bis(2-methyl-5-(4-undecyloxyphenyl)3-thienyl)perfluorocyclopentene (1a) (Scheme 1). Upon UV light irradiation, the twin crystal of 1a exhibits the different velocities in the crystal bending process even under the respective irradiation on the front and back of the crystal, which is not able to explain by the conventional diarylethene bending mechanism previously. Herein, the possible mechanism of the twin crystal bending process is discussed based on the rate of

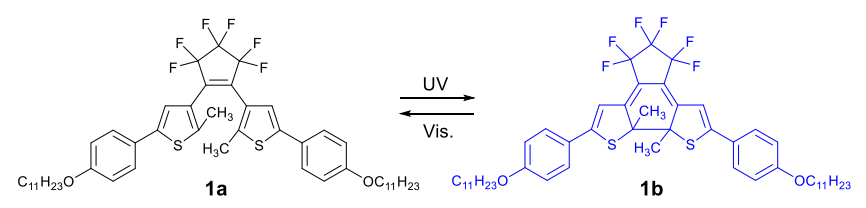

Scheme 1. Molecular structure of diarylethene 1a used in this work. 
twin components, absorption anisotropy of the diarylethene open-ring isomer and the degree of crystal twisting.

\section{Results and discussion}

\section{Photochromism in solution}

Diarylethene 1a underwent reversible photochromic reactions upon alternating irradiation with UV and visible light. Fig. 1 shows the absorption spectral change of $1 \mathbf{a}$ in $n$-hexane by photoirradiation. Upon irradiation with $313 \mathrm{~nm}$ light, the colourless solution changed to blue one. The visible absorption maximum was observed at $582 \mathrm{~nm}$ for $\mathbf{1 b}$. The absorption spectral change is based on photoisomerization from $\mathbf{1 a}$ to $\mathbf{1 b}$. The blue colour solution returned to the initial colourless solution upon irradiation with visible light $(>500 \mathrm{~nm})$. Both $1 \mathrm{1a}$ and $\mathbf{1 b}$ were stable at room temperature. It indicates that $\mathbf{1 a}$ exhibits a P-type photochromic reaction. The $\varepsilon$ values were estimated to be $46700 \mathrm{M}^{-1} \mathrm{~cm}^{-1}$ at $290 \mathrm{~nm}$ for $1 \mathrm{a}$ and 23500 $\mathrm{M}^{-1} \mathrm{~cm}^{-1}$ at $582 \mathrm{~nm}$ for $\mathbf{1 b}$ in $n$-hexane. The photocyclization and cycloreversion quantum yields were determined to be 0.55 (upon irradiation with $313 \mathrm{~nm}$ light) and 0.0033 (upon irradiation with $582 \mathrm{~nm}$ light), respectively. The photoisomerization conversion ratio from $\mathbf{1 a}$ to $\mathbf{1 b}$ was determined to be $98 \%$ in $n$-hexane upon irradiation with 313 nm light.

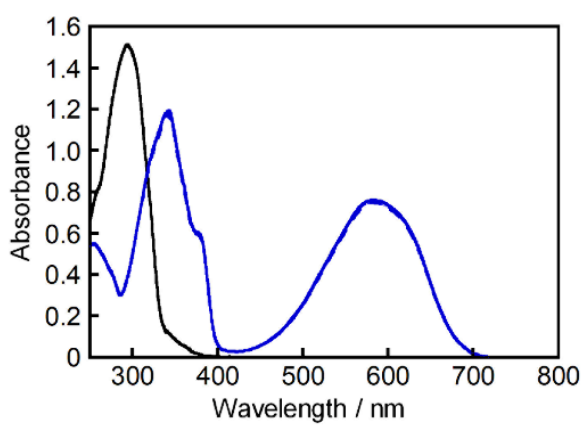

Fig. 1 UV-Vis. absorption spectral change of 1a in $n$-hexane upon photoirradiation: 1a (black line), $\mathbf{1 b}$ (blue line), and the solution in the photostationary state upon irradiation at $313 \mathrm{~nm}$ (blue broken line).

\section{Photochromism in crystal}

A rod-like crystal was obtained by recrystallization of diarylethene 1a from a mixed solvent of acetone and ethanol using a slow evaporation method under room temperature. Crystal 1a has a triclinic crystal system and a space group of $P \overline{1}$ as can be seen from $X$-ray crystallographic analysis, summarized in Table S1. All 1a molecules exist in an antiparallel conformation in the crystal. The distance between the reactive carbon atoms was estimated to be 3.729(4) A. This means that it is enough short to undergo a photocyclization reaction even in the crystalline phase. To determine molecular orientation, the crystalline nature of a well-developed face for crystal 1a was confirmed by an X-ray diffraction measurement. The diffraction peak positions corresponding to Miller planes such as (002), (003) and (005) based on the diffraction data agreed well with the calculated pattern in Fig. S1. This means that the welldeveloped face is the (001) face. Notably, it was confirmed that crystal 1a was a "twin crystal" by single crystal X-ray crystallographic analysis. In the process of a data collection and a cell refinement of diffraction spots by software as shown in Fig. S2, it was found that there were two types of spots assigned to two different components and that the unit cell of one component was consistent with that of the other component by rotating it by $180^{\circ}$ with respect to the (001) face. Thus, the twin crystal is made from two domains stuck together at the (001) face. The twin-component ratio depends on the individual twin crystal. Fig. 2 shows the shape of the twin crystal, the scanning electron microscope (SEM) image of the crystal tip, and the molecular packing diagrams. As can be seen from the SEM observation, the twin crystal is composed of two components and has a striation at the boundaries. The absorption anisotropy of the closed-ring form at $595 \mathrm{~nm}$ viewed on the (001) face is shown in Fig. S3. This vivid dichroism confirms that 1a underwent the photochromic reaction in the crystalline phase.
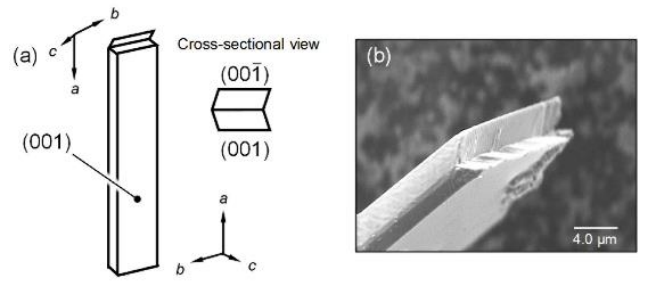

(c) Viewed from (001) face
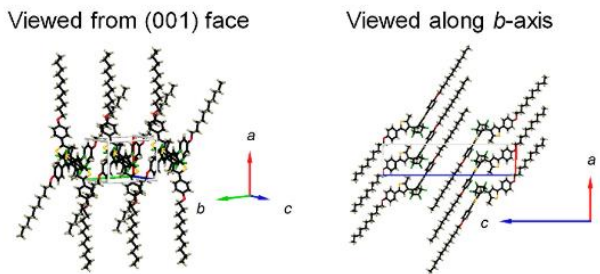

Fig. 2 (a) Shape of twin crystal consisting of 1a, (b) SEM image of the crystal tip, and (c) molecular packing diagrams viewed from (001) face and viewed along $b$-axis.

\section{Photomechanical behaviour}

A shape change of crystal 1a under photoirradiation was investigated. The size of the rod-like twin crystal is $826 \mu \mathrm{m} \times 22$ $\mu \mathrm{m} \times 12 \mu \mathrm{m}$ (length $\times$ width $\times$ thickness) before photoirradiation. The crystal was adhered to the tip of a glass capillary and observed by a CCD video camera attached to a digital microscope. When the crystal was irradiated from the (001) face and the (001) face with UV light (wavelength: $365 \mathrm{~nm}$, time: $10 \mathrm{~s}$, power: $91 \mathrm{~mW} \mathrm{~cm}^{-2}$ ), the crystal bent toward the incident light source but the bending velocity was clearly different as can be seen from Video S1, S2 (i.e. photoirradiation from the left or right side). This bending process cannot be explained by the bending mechanism of the conventional diarylethenes because photoisomerization of a diarylethene occurs on the vicinity of the crystal surface and causes the contraction or expansion along a crystal long axis based on the anisotropic molecular size change accompanied by photoirradiation. 
(a)

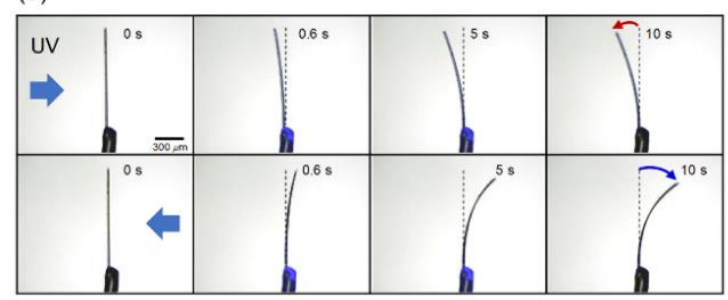

(b)

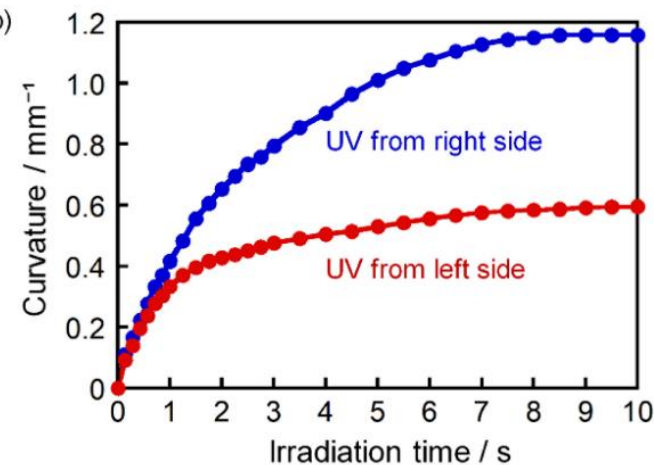

Fig. 3 Photoinduced anisotropic bending behaviour of crystal 1a (twin-component ratio: 3.0:9.0 in $\mu \mathrm{m}$ unit) upon irradiation with $365 \mathrm{~nm}$ light (power: $91 \mathrm{~mW} \mathrm{~cm}^{-2}$ ) from the left and right sides: (a) Snapshots of photoirradiated bent crystal at each time, (b) plots of curvature estimated from the snapshots.
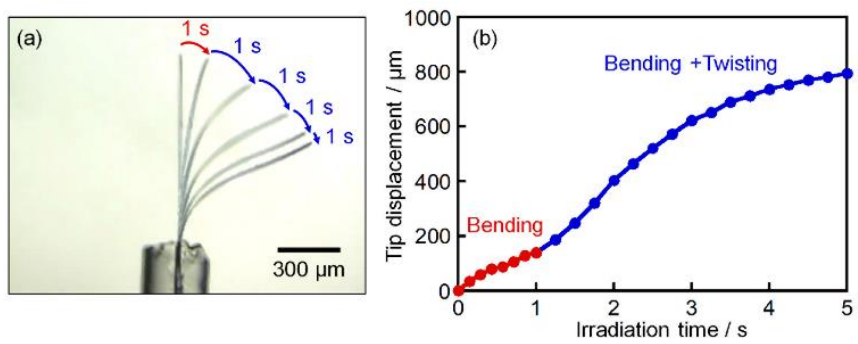

Fig. 4 Twin crystal showing bending and twisting upon irradiation with UV light from the right side: (a) the photograph showing a superimposition of 6 frames taken at intervals of $1 \mathrm{~s}$, (b) the tip displacement against UV irradiation time.
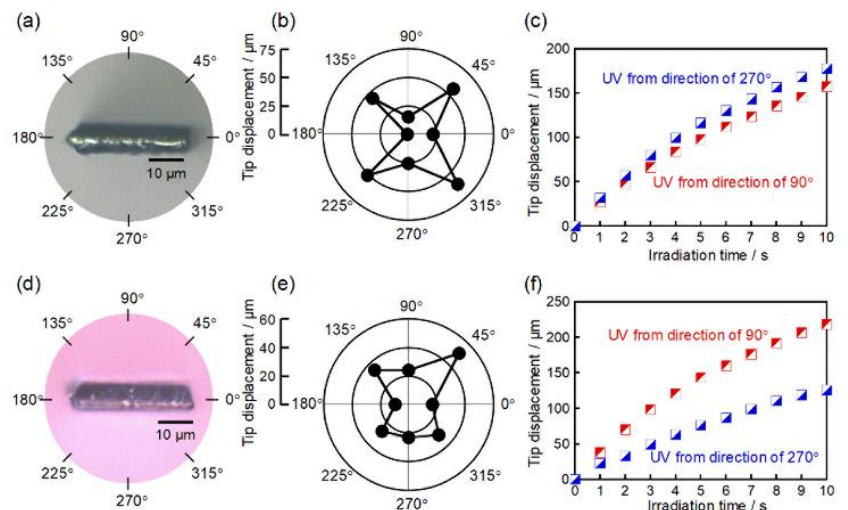

Fig. 5 The cross-section of twin crystals viewed from the tip of the crystal (a: symmetric type crystal, d: asymmetric type crystal), polar plots of the tip displacement against the incident light angle after UV irradiation for $0.82 \mathrm{~s}$ (b: symmetric type crystal, e: asymmetric type crystal), and changes in the tip displacement to irradiation time with UV light from the direction of $90^{\circ}$ and $270^{\circ}$ (c: symmetric type crystal, f: asymmetric type crystal).
Fig. 3 shows a change in the curvature of the crystal against irradiation time. In the initial irradiation time, no difference in the degree of curvature depending on the illumination directions was observed. However, as irradiation time goes on, the crystal showed the bending accompanied with twisting to result in different curvatures by the irradiation directions from the left and right sides.

The shape change of the twin crystal was observed as the tip displacement (Fig. 4 and Video S3). Within 1 s of the irradiation, the crystal showed a normal bending. The twisting behaviour was initiated after the irradiation for $1 \mathrm{~s}$.

To clarify the difference in bending velocity, we monitored the tip of the twin crystal. Two twin crystals with the different component ratio were prepared and viewed from the tip. One is the twin crystal (component ratio: 4.7:5.1 in $\mu \mathrm{m}$ unit) consisting of the inverted two single crystals symmetrically against a striation (Fig. 5a). The other consists asymmetrically (component ratio: 4.7:1.7 in $\mu \mathrm{m}$ unit) (Fig. $5 \mathrm{~d}$ ).

Fig. 5b,e show the polar plots of the tip displacement against an incident light angle with UV irradiation counterclockwise every $45^{\circ}$ from 8 directions. To observe an initial bending velocity, the tip displacement after irradiation for $0.82 \mathrm{~s}$ was plotted. When irradiated with UV light from the diagonal directions of $45^{\circ}, 135^{\circ}, 225^{\circ}$ and $315^{\circ}$, the tip moved faster. Fig. $5 \mathrm{c}$ shows the tip displacement upon irradiation with UV light from the directions of $90^{\circ}$ and $270^{\circ}$ using the symmetrical crystal. There was no difference in the tip displacement depending on the illumination directions. On contrary, in the case of the asymmetrical crystal, there was an apparent difference in the tip displacement depending on the illumination directions, as shown in Fig. 5f. The UV irradiation to the crystal side with a large component ratio moved faster than that to the side with a small component ratio. The degree of the crystal twisting based on the diagonal contraction of the crystal according to the photoisomerization from $\mathbf{1 a}$ to $\mathbf{1 b}$ was concerned with the ratio of twin compositions (Fig. S4, S5).

\section{Polarized absorption spectra of the open-ring form in crystal 1a}

To check the relationship between the photomechanical behaviour and an absorption anisotropy of the open-ring form, we examined the polarized absorption spectra. Fig. $6 \mathrm{~b}$ shows absorbance of crystal 1a at $415 \mathrm{~nm}$, which is the absorption edge, viewed on the (001) face. The absorbance at $365 \mathrm{~nm}$ is not appropriate for spectrum measurement because its absorbance is too large. Thus, the absorption anisotropy was measured at the absorption edge at $415 \mathrm{~nm}$ (Fig. S6). The absorbance at the absorption edge was the largest when crystal 1a was rotated at $\vartheta=0^{\circ}$ and $180^{\circ}$, and it was a minimum at $\vartheta=$ $90^{\circ}$ and $270^{\circ}$. The absorption anisotropy of 1 a did not correlate with that of $\mathbf{1 b}$ in Fig. S3. 

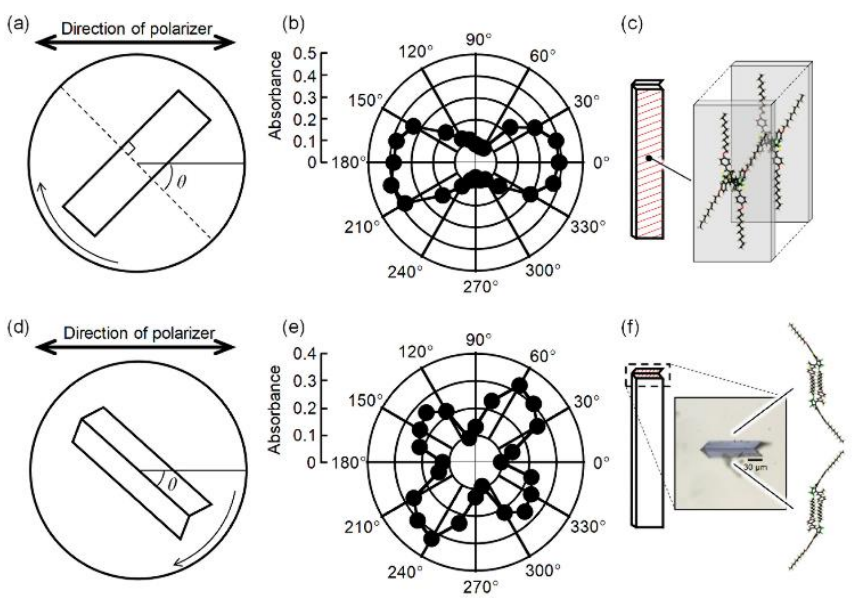

Fig. 6 The direction of the polarizer (a: the flat plane of the (001) face, d: the tip of the twin crystal), polar plots of absorbance of $1 \mathrm{a}$ at $415 \mathrm{~nm}$ for crystal 1a (b: (001) face, e: the tip), and molecular packing of crystal $1 a$ (c: viewed from (001) face, f: viewed from the tip).

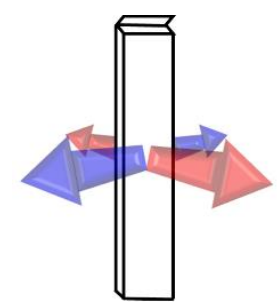

Fig. 7 Schematic illustration of the electronic transition moments of 1 a in the twin crystal represented by red and blue arrows.

Fig. 6e shows the polar plot for absorbance of crystal 1a at $415 \mathrm{~nm}$ viewed from the crystal tip. Polarized absorption spectra of the crystal tip were measured by cutting the cross section of the twin crystal with a razor and standing it on a glass slide. The molecular absorption coefficient was large when viewed from the directions of $45^{\circ}, 135^{\circ}, 225^{\circ}$ and $315^{\circ}$, and it was small on the directions of $90^{\circ}$ and $270^{\circ}$.

Hence, it was found that the photoinduced bending velocity of the crystal was deeply correlated with the absorption anisotropy of 1a. The absorption anisotropy of the twin crystal depends on the ratio of the twin components. To make it clear to see, Fig. 7 shows the schematic illustration of the absorption anisotropy of 1a. These red and blue arrows represent the direction of the electronic transition moment of 1 a. This indicates that the light absorption from the diagonal directions such as $45^{\circ}, 135^{\circ}, 225^{\circ}$ and $315^{\circ}$ is advantageous. Based on these results, we propose schematic illustrations for mechanism of the photoinduced crystal bending with different velocities depending on the illumination directions, as shown in Fig. 8.
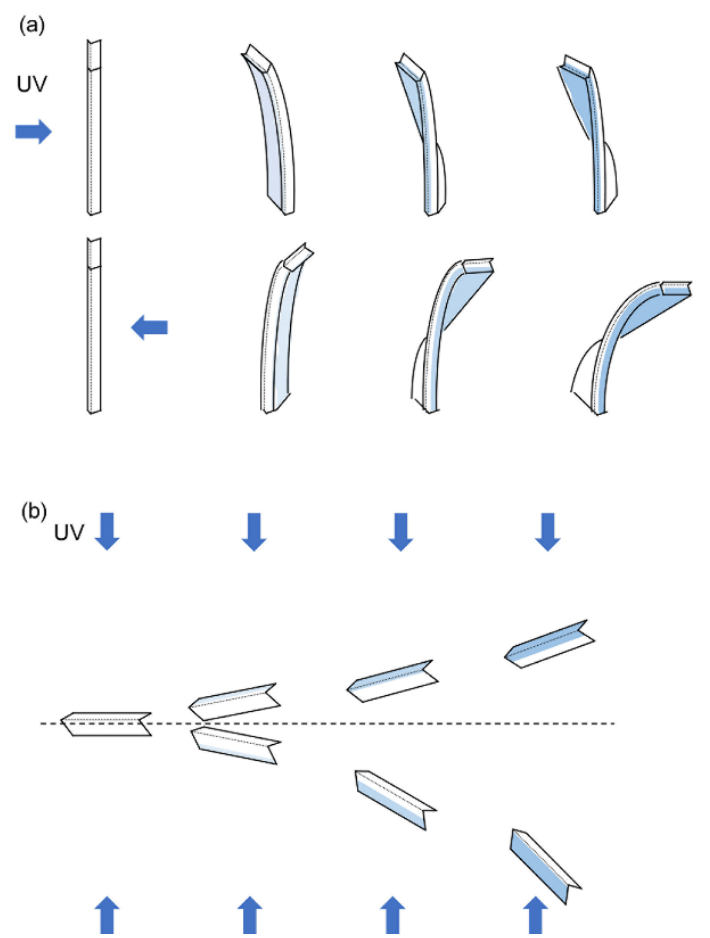

Fig. 8 Schematic illustrations of the photoinduced bending mechanism with different velocities depending on the illumination directions: (a) viewed from the front, (b) viewed from the top.

This mechanical work occurs in the twin crystal having the ratio of different components. Upon UV light irradiaton, the crystal side with a large component moves faster than the side with a small one. In the initial irradiation process, the crystal bending velocities were almost the same even in the irradiation to (001) and $(00 \overline{1})$ faces. However, as the irradiation time proceeded, the twin crystal gradually twisted due to the diagonal contraction of the anisotropic size change of the molecule 1a. The crystal twisting takes place in both a lefthanded helix and a right-handed helix. The direction of the twisting depends upon the face irradiated with UV light. The contraction of the unit cell of the (001) face takes place in the direction of top-right to bottom-left when UV light was irradiated to the (001) face. On the other hand, the contraction of the unit cell of the (001) face takes place in the direction of top-left to bottom-right when UV light was irradiated to the (00 1) face. Therefore, the direction of twisting (the left-handed helix versus the right-handed helix) can be determined by the irradiation face. When the photochromic reaction crosses the striation to the other side, the crystal twisting is suppressed due to the inverted molecular packing of the twin components. As a results, the movement of the crystal twisting is suppressed by irradiation from the side with a small component ratio, and it twists well by irradiation from the side with a large one. The degree of twisting depends on the ratio of twin components by photoreaction depth beyond the striation. As the crystal twisting goes by, the irradiation surface is changed, it becomes the same irradiation condition in the case upon irradiation with UV light from the direction to the crystal face with a high molecular absorption coefficient (i.e. upon irradiation with UV 
light from diagonal direction). Hence, the movement in the crystal bending is accelarated because light absorption is advantage from diagonal direction, resulting in the difference in the crystal bending velocity. The difference in the molecular absorption coefficient is explained by the absorption anisotropy of the diarylethene open-ring form in the crystal. Hence, the difference of the photoinduced crystal bending behaviour with twisting depending on the illumination directions can be explained by the ratio of the different components and the absorption anisotropy of $\mathbf{1 a}$.

\section{Conclusion}

The photoinduced shape change of the twin crystal composed of diarylethene 1a was investigated. It was confirmed that the rod-like crystal 1a obtained by recrystallization consisted of the twin crystal. Crystal 1a showed the different crystal bending velocities when irradiated to the (001) and (001) faces. In the initial irradiation time, the crystal bending velocities were not much different, however, as time passed, the difference was large. The tip displacement against the incident light angle was markedly large when irradiated with UV light from diagonal directions. It was found that the difference in the velocity of the crystal bending deeply correlated with the ratio of the different components. Furthermore, it was found that the anisotropy of the crystal bending velocities corresponds to the absorption anisotropy of 1a. We proposed the comprehensive mechanism of the photomechanical behaviour of the twin crystal, which is ascribed to the ratio of the twin components, the absorption anisotropy of 1a and the degree of crystal twisting.

\section{Experimental section}

\section{General}

Solvents used were spectroscopic grade and purified by distillation before use. ${ }^{1} \mathrm{H}$ NMR $(300 \mathrm{MHz})$ and ${ }^{13} \mathrm{C}$ NMR (75 $\mathrm{MHz}$ ) spectra were recorded on a Bruker AV-300N spectrometer with tetramethylsilane (TMS) as the internal standard. Mass spectra were obtained on a JEOL JMS-700/700S mass spectrometer. Absorption spectra were measured with a JASCO V-560 absorption spectrometer. Photoirradiation in solution was conducted using a $200 \mathrm{~W}$ mercury-xenon lamp (Moritex MUV-202) or a 300 W xenon lamp (Asahi Spectra MAX301) as a light source. Monochromatic light was obtained by passing the light through a monochromator (Jobin Yvon H10UV) and glass filters. Photocyclization and photocycloreversion quantum yields were determined in $n$-hexane as relative quantum yields using typical diarylethene, whose quantum yields have already been reported. The photocyclization conversion ratio was determined by absorption spectroscopic analysis. The image of a twin crystal was observed by a Keyence VE-7800 scanning electron microscopy. The bending behaviour of crystals was observed using a Keyence VHX-500 digital microscope. Polarized absorption spectra were measured using a Nikon ECLIPSE E600POL polarizing optical microscope connected with a Hamamatsu PMA-11 photodetector. Powder
$X$-ray diffraction profiles were recorded on a Rigaku MiniFlex 600 diffractometer employing $\mathrm{CuK}_{\alpha}$ radiation $(\lambda=1.54184 \AA$ ) . Single crystal $\mathrm{X}$-ray crystallographic analysis was carried out using a Rigaku VariMax with Saturn CCD diffractometer with MoK $_{\alpha}$ radiation $(\lambda=0.71073 \AA$ ) monochromated by the graphite. The crystal structures were solved by a direct method using SIR92 and refined by the full-matrix least-squares method on $F^{2}$ with anisotropic displacement parameters for non-hydrogen atoms using SHELXL-2014. UV irradiation to the crystal was carried out using a Keyence UV-LED UV-400 (365 nm light) or a super high-pressure mercury lamp (100 W; UV-1A filter (365 nm light excitation)) attached to the polarizing optical microscope. The light power was measured using a Neoark PM-335A power meter. Visible light irradiation was carried out using a halogen lamp (100 W).

\section{Materials}

1,2-Bis(2-methyl-5-(4-undecyloxyphenyl)-3-thienyl)perfluorocyclopentene (1a) was synthesized as follows: To $55 \mathrm{~mL}$ of dry THF solution containing 3-Bromo-2-methyl-5-(4-undecyloxyphenyl)thiophene $(1.2 \mathrm{~g}, 3.0 \mathrm{mmol})$ was added $2.1 \mathrm{~mL}$ of $1.6 \mathrm{M}$ $n$-BuLi hexane solution $(3.3 \mathrm{mmol})$ at $-78{ }^{\circ} \mathrm{C}$ under argon atmosphere, and the solution was stirred for $1.5 \mathrm{~h}$ at $-40{ }^{\circ} \mathrm{C}$. Octafluorocyclopentene $(0.20 \mathrm{~mL}, 1.5 \mathrm{mmol}$, Nippon Zeon) was slowly added to the reaction mixture at $-78^{\circ} \mathrm{C}$, and the mixture was stirred for $5 \mathrm{~h}$ at that temperature. The reaction was stopped by the addition of water. The product was extracted with ether. The organic layer was dried over $\mathrm{MgSO}_{4}$, filtrated, and concentrated. The residue was purified by column chromatography on silica gel using $n$-hexane/ethyl acetate (95:5), recycling preparative HPLC on polystyrene gel using chloroform as the eluent, and HPLC on silica gel using $n$ hexane/ethyl acetate (95:5) as the eluent to give $492 \mathrm{mg}$ of 1a in $38 \%$ yield. Finally, 1a was purified by recrystallization from a mixed solvent of acetone and ethanol. 1a: $\mathrm{mp}: 79.4-80.3^{\circ} \mathrm{C} .{ }^{1} \mathrm{H}$ NMR (300 MHz, $\left.\mathrm{CDCl}_{3}\right): \delta 0.88(\mathrm{t}, J=6.7 \mathrm{~Hz}, 6 \mathrm{H}), 1.2-1.5(\mathrm{~m}$, $32 \mathrm{H}), 1.7-1.9(\mathrm{~m}, 4 \mathrm{H}), 1.94(\mathrm{~s}, 6 \mathrm{H}), 3.97(\mathrm{t}, J=6.6 \mathrm{~Hz}, 4 \mathrm{H}), 6.90$ (d, $J=8.7 \mathrm{~Hz}, 4 \mathrm{H}$ ), 7.15 (s, 2H), 7.45 (d, $J=8.7 \mathrm{~Hz}, 4 \mathrm{H}) .{ }^{13} \mathrm{C} \mathrm{NMR}$ $\left(75 \mathrm{MHz}, \mathrm{CDCl}_{3}\right.$ ): $\delta 14.3,14.6,22.8,26.2,29.4,29.5,29.7,29.8$, $32.1,68.3,115.1,121.3,125.9,126.1,127.0,140.3,142.3,159.2$ HRMS(FAB): $m / z=860.4097\left(\mathrm{M}^{+}, 100 \%\right)$, Calcd for $\mathrm{C}_{49} \mathrm{H}_{62} \mathrm{~F}_{6} \mathrm{O}_{2} \mathrm{~S}_{2}$ $=860.4095$.

\section{Author contributions}

Takuya Higashiguchi: Data curation, Formal analysis, Investigation, Methodology, Visualization, Writing - original draft; Daichi Kitagawa: Formal analysis, Methodology, Visualization, Writing - original draft, Writing - review \& editing; Seiya Kobatake: Conceptualization, Methodology, Project administration, Resources, Supervision, Visualization, Writing review \& editing.

\section{Conflicts of interest}

There are no conflicts to declare. 


\section{Acknowledgements}

The authors also thank Nippon Zeon Co., Ltd. for providing octafluorocyclopentene.

\section{Notes and references}

1 J. Lee, S. Oh, J. Pyo, J.-M. Kim and J. Ho Je, Nanoscale, 2015, 7, 6457-6461.

2 R. O. Al-Kaysi, F. Tong, M. Al-Haidar, L. Zhu and C. J. Bardeen, Chem. Commun., 2017, 53, 2622-2625.

3 Q. Yu, X. Yang, Y. Chen, K. Yu, J. Gao, Z. Liu, P. Cheng, Z. Zhang, B. Aguila and S. Ma, Angew. Chem. Int. Ed., 2018, 57, 1019210196.

4 S. Kobatake, S. Takami, H. Muto, T. Ishikawa and M. Irie, Nature, 2007, 446, 778-781.

5 M. Morimoto and M. Irie, J. Am. Chem. Soc., 2010, 132, 417214178.

6 D. Kitagawa and S. Kobatake, Chem. Commun., 2015, 51, 4421-4424.

7 F. Terao, M. Morimoto and M. Irie, Angew. Chem. Int. Ed., 2012, 51, 901-904.

8 D. Kitagawa and S. Kobatake, J. Phys. Chem. C, 2013, 117 20887-20892.

9 D. Kitagawa, R. Tanaka and S. Kobatake, Phys. Chem. Chem. Phys., 2015, 17, 27300-27305.

10 A. Hirano, T. Hashimoto, D. Kitagawa, K. Kono and S. Kobatake, Cryst. Growth Des., 2017, 17, 4819-4825.

11 A. Hirano, D. Kitagawa and S. Kobatake, CrystEngComm, 2019 21, 2495-2501.

12 D. Kitagawa, H. Tsujioka, F. Tong, X. Dong, C. J. Bardeen and S. Kobatake, J. Am. Chem. Soc., 2018, 140, 4208-4212.

13 D. Kitagawa, R. Tanaka and S. Kobatake, CrystEngComm, 2016, 18, 7236-7240.

14 D. Kitagawa, K. Kawasaki, R. Tanaka and S. Kobatake, Chem. Mater., 2017, 29, 7524-7532.

15 D. Kitagawa, H. Nishi and S. Kobatake, Angew. Chem. Int. Ed., 2013, 52, 9320-9322.

16 K. Uchida, S. Sukata, Y. Matsuzawa, M. Akazawa, J. J. D. de Jong, N. Katsonis, Y. Kojima, S. Nakamura, J. Areephong, A. Meetsma and B. L. Feringa, Chem. Commun., 2008, 326-328.

17 D. Kitagawa, T. Okuyama, R. Tanaka and S. Kobatake, Chem. Mater., 2016, 28, 4889-4892.

18 K. Uchida, N. Izumi, S. Sukata, Y. Kojima, S. Nakamura and M. Irie, Angew. Chem. Int. Ed., 2006, 45, 6470-6473.

19 K. Uchida, N. Nishikawa, N. Izumi, S. Yamazoe, H. Mayama, Y. Kojima, S. Yokojima, S. Nakamura, K. Tsujii and M. Irie, Angew. Chem. Int. Ed., 2010, 49, 5942-5944.

20 E. Hatano, M. Morimoto, T. Imai, K. Hyodo, A. Fujimoto, R. Nishimura, A. Sekine, N. Yasuda, S. Yokojima, S. Nakamura and K. Uchida, Angew. Chem. Int. Ed., 2017, 56, 12576-12580.

21 H. Koshima, N. Ojima and H. Uchimoto, J. Am. Chem. Soc., 2009, 131, 6890-6891.

22 T. Taniguchi, J. Fujisawa, M. Shiro, H. Koshima and T. Asahi, Chem.-Eur. J., 2016, 22, 7950-7958.

23 O. S. Bushuyev, T. C. Corkery, C. J. Barrett and T. Friščić, Chem. Sci., 2014, 5, 3158-3164.

24 O. S. Bushuyev, A. Tomberg, T. Friščić and C. J. Barrett, J. Am. Chem. Soc., 2013, 135, 12556-12559.

25 T. Taniguchi, H. Sugiyama, H. Uekusa, M. Shiro, T. Asahi and H. Koshima, Nat. Commun., 2018, 9, 538.

26 H. Koshima, K. Takechi, H. Uchimoto, M. Shiro and D. Hashizume, Chem. Commun., 2011, 47, 11423-11425.

27 Y. Hagiwara, T. Taniguchi, T. Asahi and H. Koshima, J. Mater. Chem. C, 2020, 8, 4876-4884.

28 H. Koshima, H. Nakaya, H. Uchimoto and N. Ojima, Chem. Lett., 2012, 41, 107-109.
29 L. Zhu, R. O. Al-Kaysi and C. J. Bardeen, J. Am. Chem. Soc., 2011 133, 12569-12575.

30 R. O. Al-Kaysi, A. M. Müller and C. J. Bardeen, J. Am. Chem. Soc., 2006, 128, 15938-15939.

31 T. Kim, L. Zhu, L. J. Mueller and C. J. Bardeen, J. Am. Chem. Soc., 2014, 136, 6617-6625.

32 F. Tong, M. Al-Haidar, L. Zhu, R. O. Al-Kaysi and C. J. Bardeen, Chem. Commun., 2019, 55, 3709-3712.

33 Y. Matsunaga, K. Goto, K. Kubono, K. Sako and T. Shinmyozu, Chem.-Eur. J., 2014, 20, 7309-7316.

34 M. Irie, T. Fukaminato, K. Matsuda and S. Kobatake, Chem. Rev., 2014, 114, 12174-12277.

35 S. Timoshenko, J. Opt. Soc. Am., 1925, 11, 233-255.

36 P. Naumov, S. Chizhik, M. K. Panda, N. K. Nath and E. Boldyreva, Chem. Rev., 2015, 115, 12440-12490. 